02,11

\title{
Критический ток фазового перехода в тонких пленках нитрида ниобия
}

\author{
(C) М.А. Васютин, Н.Д. Кузьмичев, Д.А. Шилкин \\ Мордовский государственный университет им. Н.П. Огарёва, \\ Саранск, Россия \\ E-mail: vasyutinm@mail.ru
}

(Поступила в Редакцию 21 мая 2018 г.)

Исследовано влияние тока и магнитного поля на переход в сверхпроводящее состояние в тонких пленках нитрида ниобия. Определены температурные и магнитополевые зависимости критического тока пленок в области перехода. В рамках моделей нормального домена и разогрева квазичастиц при вязком течении магнитного потока найдены функциональные зависимости критического тока в непосредственной близости к температуре перехода в сверхпроводящее состояние. Из теории разогрева электронов определено время их энергетической релаксации. Оценены время электрон-фононного и электрон-электронного неупругого рассеяний, коэффициент диффузии электронов, коэффициент теплоотвода.

DOI: 10.21883/FTT.2018.11.46671.138

\section{1. Введение}

Нитрид ниобия $(\mathrm{NbN})$ обладает физическими свойствами, которые используются как в прикладной, так и в фундаментальной науке. Пленки из $\mathrm{NbN}$ отличаются простотой изготовления, химической и радиационной устойчивостью, механической прочностью и высоким вторым критическим полем. В прикладной физике в последнее время активно изучаются низкоразмерные структуры на основе нитрида ниобия, применяющиеся в качестве болометров в радиоастрономии и терагерцовой спектроскопии (hot-electron bolometer, HEB) [1]. Тонкие пленки из $\mathrm{NbN}$ используются в качестве однофотонных детекторов (superconducting single-photon detectors, SSPDs) [2]. Недавно в нитриде ниобия был обнаружен обратный спиновый эффект Холла [3], перспективный для спинтроники.

Для фундаментальной физики исследование нитрида ниобия также актуально, например, в связи с изучением перехода сверхпроводник-изолятор в ультратонких пленках $\mathrm{NbN}$ [4]. Результаты этой работы проясняют картину перехода Березинского-Костерлица-Таулеса (БКТ) в высокотемпературных сверхпроводниках (ВТСП). Вблизи квантовой критической точки энергия вихревого кора оказалась связанной с энергетической щелью, а не со сверхтекучей плотностью. БКТ-физика в купратных ВТСП (в частности, в $\mathrm{YBa}_{2} \mathrm{Cu}_{3} \mathrm{O}_{7-x}$ $(\mathrm{YBCO}))$ усложняется из-за малой длины когерентности в них, что затрудняет наблюдение БКТ-перехода, происходящего несогласованно в отдельных $\mathrm{CuO}_{2}$-слоях (в работе [5] подтверждено наличие джозефсоновских плазмонов между $\mathrm{CuO}_{2}$-слоями, свидетельствующих о слабости межслоевого взаимодействия). Нитрид ниобия близок к YВСО по концентрации носителей, длине когерентности и глубине проникновения магнитного поля [6,7]. Поэтому изучение пленок $\mathrm{NbN}$ может помочь в понимании механизма разрушения сверхпроводимости током, магнитным полем и тепловыми вихрями вблизи температуры перехода в сверхпроводящее состояние $\left(T_{c}\right)$ в YВСО [8-11].

Чрезвычайно интересным явилось и открытие моды Хиггса в $\mathrm{NbN}[12,13]$, что очень важно для физики элементарных частиц. Кроме этого, в работе [14] был обнаружен своеобразный конфайнмент сверхпроводящих флуктуаций, объясняющий сосуществование псевдощели и нульмерных флуктуаций амплитуды параметра порядка в нитриде ниобия.

Пленки, изучаемые в данной статье, довольно сильно отличаются от исследованных в других работах сочетанием высокой температуры перехода и высоким удельным сопротивлением в нормальном состоянии $\rho_{n}$. Увеличение удельного сопротивления в нашем случае не приводит к уменьшению $T_{c}[15]$, так как нитрид ниобия является $s$-волновым сверхпроводником. Сопротивление должно расти с ростом беспорядка, но величина $T_{c}$, подавляемая в этом случае, зависит и от плотности состояний на уровне Ферми.

Исследование температурной и магнитополевой зависимостей критического тока перехода в резистивное состояние $I_{\mathrm{cp}}(T, H)$ является продолжением изучения пленок $\mathrm{NbN}$, начатым в работе [16], где определялась температурная зависимость второго критического магнитного поля данных образцов. Функциональные зависимости $I_{\mathrm{cp}}(T, H)$ содержат информацию о динамике вихревого и доменного состояний пленок, параметрах электрон-фононного взаимодействия.

\section{2. Теория}

Критический ток является структурно-чувствительным параметром сверхпроводника, что должно учитываться при анализе его температурных и магнитополевых зависимостей. Принимая во внимание наличие неоднородностей в исследуемых пленках в виде границ 
зерен и близость к $T_{c}$, для описания экспериментальных зависимостей можно применить одномерную модель возникновения нормального домена в сверхпроводящей пленке [17] (токовые и потенциальные контакты были сформированы по всей ширине образца).

Пленка сверхпроводника моделируется системой сверхпроводник-нормальный участок-сверхпроводник. Нормальный участок $(\mathrm{N})$ с обеих сторон контактирует со сверхпроводящими участками $(\mathrm{S})$. NS-граница под действием джоулева тепла при протекании тока может сдвигаться, образуя резистивную область в сверхпроводнике (нормальный домен).

Уравнение стационарной теплопроводности, описывающее распределение температуры $T$ вдоль пленки при заданной плотности тока $J$, имеет вид [17]

$$
-\kappa(x) \frac{d^{2} T}{d x^{2}}+\alpha(x)\left(T-T_{0}\right)=\rho(x) J^{2},
$$

где $\kappa-$ коэффициент теплопроводности, $\alpha-$ коэффициент теплоотвода, $\rho$ - удельное сопротивление пленки, $T_{0}$ - температура окружающей среды. Коэффициенты, входящие в уравнение (1), предполагаются не зависящими от температуры кусочно-непрерывными функциями.

Решая уравнение (1) при условии равенства критической температуры и температуры NS-границы в заданном магнитном поле с магнитной индукцией $B$, для тока перехода пленки в нормальное состояние $I_{\mathrm{cp}}$ можно получить [17]

$$
I_{\mathrm{cp}}\left(B, T_{0}\right)=I_{0} \sqrt{1-\frac{T_{0}}{T_{c 0}}} \sqrt{\frac{1-\frac{\left(\frac{d H_{c 2}}{d T}\right)_{T_{c 0}}\left(T_{c 0}-T_{0}\right)}{1+\frac{B}{\left(\frac{d H_{c 2}}{d T}\right)_{T_{c 0}}\left(T_{c 0}-T_{0}\right)}}}{2}}
$$

где $I_{\mathrm{cp}}\left(B, T_{0}\right)=I_{\mathrm{eq}}$ - ток равновесия NS-границы, $T_{c 0}-$ температура перехода в сверхпроводящее состояние в нулевом магнитном поле, $I_{0}=\left(2 \alpha_{\mathrm{nd}} T_{c 0} / \rho_{\mathrm{nd}}\right)^{1 / 2} d w, d-$ толщина пленки, $w$ - ширина пленки, $\left(d H_{c 2} / d T\right)_{T_{c 0}}-$ производная второго критического поля по температуре при $T=T_{c 0}, \alpha_{\mathrm{nd}}$ и $\rho_{\mathrm{nd}}-$ коэффициент теплоотвода и удельное сопротивление нормального домена.

В пленках, находящихся в магнитном поле, резистивный участок может образовываться и движением абрикосовских вихрей под действием тока. Такая модель вязкого течения магнитного потока может реализовываться в „грязных“ сверхпроводниках при учете разогрева квазичастиц $[18,19]$. Для резистивного состояния пленки, обусловленного диссипативным движением вихрей и разогревом квазичастиц, можно получить $[17,19]$

$$
I_{\mathrm{cp}}\left(T_{0}, B\right)=I_{0}^{*} \frac{\sqrt[4]{\left(1-\frac{T_{0}}{T_{c}(B)}\right)^{3}}}{\sqrt[4]{\left(1+\frac{B}{B_{T}}\right)^{3}}}
$$

где $I_{0}^{*}, B_{T}$ - параметры сверхпроводника (см. обсуждение), $T_{c}(B)$ - температура перехода в магнитном поле с индукцией $B$.

\section{3. Эксперимент и обработка результатов}

В работе изучались пленки $\mathrm{NbN}$ толщиной $d=400 \mathrm{~nm}$, шириной $w=5 \mathrm{~mm}$, длиной $a=9 \mathrm{~mm}$ на подложках из полированного плавленого кварца. Исследования проводились четырехконтактным методом при пропускании через образец переменного тока с амплитудой до $3 \mathrm{~mA}$ и частотой $1 \mathrm{kHz}$. Критический ток перехода определялся по достижению половинного значения напряжения первой гармоники сигнала отклика по сравнению с нормальным состоянием при $17 \mathrm{~K}$. Значение $T_{c}$ образцов менялось в пределах $16.2-16.5 \mathrm{~K}$. Ширина перехода $\sim 0.1 \mathrm{~K}$. Удельное сопротивление в нормальном состоянии вблизи перехода $\sim 1000 \mu \Omega \cdot \mathrm{cm}$. Подробная аттестация образцов и техника эксперимента приведены в [16]. Погрешность измерения температуры не превышала $\Delta T=0.005 \mathrm{~K}$ (датчик - Cernox CX-1050, контроллер - LakeShore 335), относительная погрешность $\delta t=\Delta T / T_{c} \approx 0.0003$.

На рис. 1 изображена магнитополевая зависимость критического тока перехода $I_{\mathrm{cp}}$ образца $\mathrm{NbN}$ с $T_{c 0}=16.480 \mathrm{~K}$ при различных температурах. Обработка экспериментальных данных сделана с помощью формул (2) и (3) с параметрами $I_{0}=32 \mathrm{~mA}$ и $I_{0}^{*}=128 \mathrm{~mA}$.

На рис. 2 показана температурная зависимость того же образца без внешнего поля и в поле $B=1 \mathrm{~T}$. Без поля аппроксимация сделана с помощью формулы (3), в магнитном поле - по формуле (2) с теми же параметрами.

\section{4. Обсуждение}

Для оценки критической плотности тока сверхпроводника $J_{c}$ можно воспользоваться простой формулой, полученной из энергетических соображений в работе [20]

$$
J_{c}=n e \Delta /\left(m v_{\mathrm{F}}\right)
$$

где $n$ - концентрация электронов проводимости, $e-$ заряд электрона, $m$ - масса электрона, $v_{\mathrm{F}}-$ фермиевская скорость электрона, $\Delta$ - энергетическая щель сверхпроводника.

Параметры наших образцов $\mathrm{NbN}: n=2.3 \cdot 10^{26} \mathrm{~m}^{-3}[6]$, $v_{\mathrm{F}}=2.2 \cdot 10^{5} \mathrm{~m} / \mathrm{s}$. Температурная зависимость щели вблизи $T_{c}[21]: \Delta(t)=1.74 \cdot \Delta(0)(1-t)^{1 / 2}, t=T_{0} / T_{c 0}$ приведенная температура, $\Delta(0)$ - энергетическая щель при $0 \mathrm{~K}$. Тогда, с учетом того, что $\Delta(0) \approx 2.5 \mathrm{meV}$ для исследуемых образцов $\mathrm{NbN}$ (в модели БКШ), получим из (4) $J_{c} \approx 8.6 \cdot 10^{5} \mathrm{~A} / \mathrm{cm}^{2}$ при $t=0.9953$. Плотность тока в наших образцах при той же температуре не превышала $10^{2} \mathrm{~A} / \mathrm{cm}^{2}$. Отличие более, чем на три порядка показывает, что в нашем случае подавление сверхпроводимости не вызвано достижением критической плотности тока.

Разрушение сверхпроводимости в исследуемых образцах может происходить из-за нагрева джоулевым 


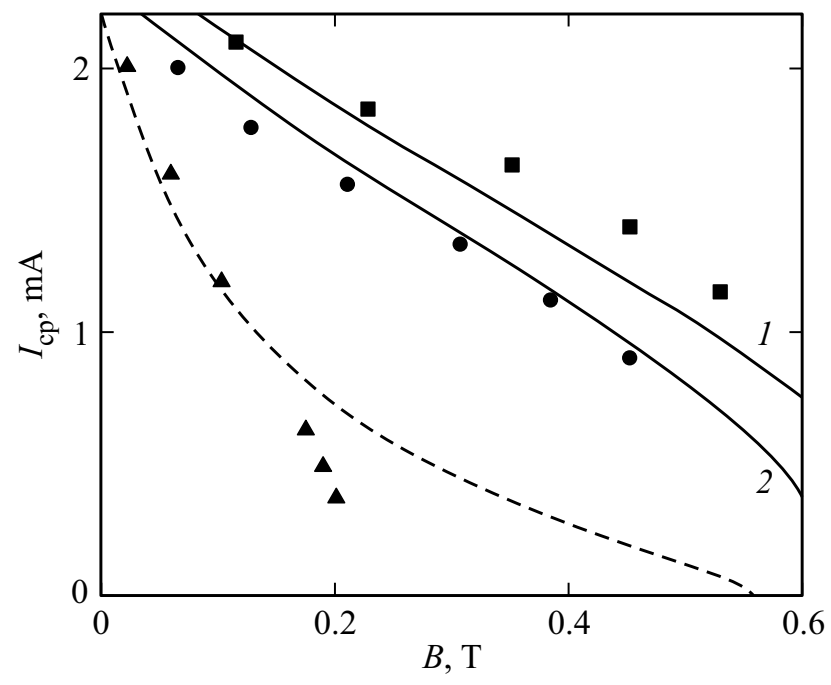

Рис. 1. Магнитополевые зависимости критического тока перехода для разных приведенных температур: (квадраты) $t_{1}=0.9940, \quad$ (кружки) $-t_{2}=0.9947, \quad$ (треугольники) $t_{3}=0.9953$. Сплошные линии - теоретические зависимости (формула (2), $\left.1-t_{1}=0.9940,2-t_{2}=0,9947\right)$, штриховая линия - теоретическая зависимость (формула (3)).

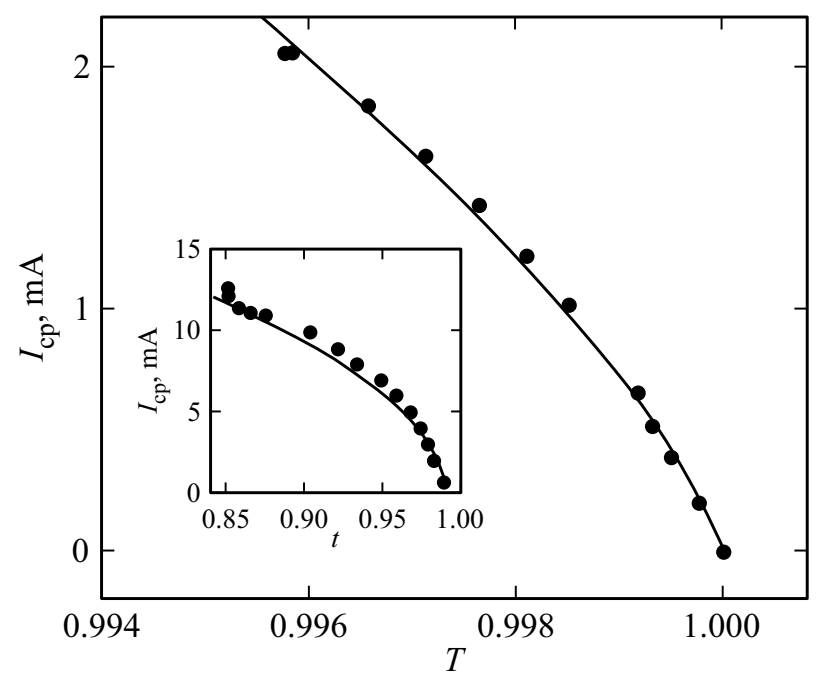

Рис. 2. Температурная зависимость критического тока перехода в нулевом магнитном поле (кружки - эксперимент, сплошная линия - теория (формула (3)). На вставке приведена температурная зависимость критического тока перехода в магнитном поле $1 \mathrm{~T}$ (кружки - эксперимент, сплошная линия - теория (формула (2)).

теплом (модель нормального домена [17]) и по причине вязкого движения вихрей и связанного с ним перегрева электронов (модель разогрева квазичастиц $[18,19]$ ).

Из рис. 1 видно, что модель нормального домена достаточно хорошо описывает $I_{\mathrm{cp}}(B)$ при температурах $t_{1}=0.9940$ и $t_{2}=0.9947$ (формула (2)), тогда как при большей температуре $t_{3}=0.9953$ требуется учет разогрева электронов при вязком течении вихрей (формула (3)).
Температурная зависимость $I_{\text {ср }}$ (рис. 2) также требует две модели для аппроксимации экспериментальных данных. В отсутствие магнитного поля применима теория разогрева электронов. В магнитном поле с индукцией $1 \mathrm{~T}$ более адекватной оказывается модель нормального домена.

Величина $I_{0}^{*}$, имеющая одно и то же значение в магнитополевых и температурных зависимостях, может служить достаточно надежным основанием для определения одного из важнейших параметров сверхпроводника времени энергетической релаксации $\tau_{\varepsilon}$. Для плотности тока $J_{0}^{*}=I_{0}^{*} /(w d)$ в работе [19] получено выражение:

$$
J_{0}^{*}=2.62 \frac{\sigma_{n}}{e} \frac{1}{\sqrt{D \tau_{\varepsilon}}} k_{\mathrm{B}} T_{c}(B) \sqrt[4]{\left(1-\frac{T_{0}}{T_{c}(B)}\right)^{3}} .
$$

где $\sigma_{n}$ - удельная проводимость образца в нормальном состоянии, $e-$ заряд электрона, $D-$ коэффициент диффузии электронов, $k_{\mathrm{B}}$ - постоянная Больцмана.

Из формулы (5) при известных значениях $D$ и $\sigma_{n}$ можно определить $\tau_{\varepsilon}$. Коэффициент диффузии для „грязного“ сверхпроводника находится по формуле [22]: $D=4 k_{\mathrm{B}} /\left(\pi e\left(-d H_{c 2} / d T\right)_{T-T_{c}}\right)$. Учитывая значение $\left.\left.-d H_{c 2} / d T\right)_{T=T_{c}}\right)=7.28 \mathrm{~T} / \mathrm{K}[16]$, получим $D \approx 0.12 \mathrm{~cm}^{2} / \mathrm{c}$. Тогда, принимая $\sigma_{n} \approx 10^{5} \mathrm{~S} / \mathrm{m}[16]$, из формулы (5) найдем $\tau_{\varepsilon}=0.9 \pm 0.5 \mathrm{~ns}$.

Время электрон-фононного взаимодействия может быть оценено по формуле [19]

$$
\tau_{\mathrm{ep}}\left(T_{0}\right)=\frac{(2 \pi)^{3} N(0) \hbar^{7} \rho_{v} s^{4}}{14 \xi(3) m^{2} \mu^{2}\left(k_{\mathrm{B}} T_{0}\right)^{3}},
$$

где $N(0)$ - плотность состояний на поверхности Ферми, $\hbar \approx 1.05 \cdot 10^{-27} \mathrm{erg} \cdot \mathrm{s}-$ постоянная Планка, $\rho_{V}-$ плотность пленки, $s$ - продольная скорость звука в пленке, $\zeta(3) \approx 1.202$ - постоянная Апери, $\mu$ - деформационный потенциал (порядка энергии Ферми).

Учитывая параметры наших образцов $(N(0) \approx$ $3.6 \cdot 10^{33} \mathrm{states} /\left(\mathrm{cm}^{3} \cdot \mathrm{erg}\right), \rho_{V} \approx 8.4 \mathrm{~g} / \mathrm{cm}^{3}, s=\left(E / \rho_{V}\right)^{1 / 2}$, здесь $E=4.8 \cdot 10^{11} \mathrm{~Pa} \quad[23]-$ модуль Юнга. $\left.S \approx 7.56 \cdot 10^{5} \mathrm{~cm} / \mathrm{c}, \quad \mu \sim \varepsilon_{\mathrm{F}} \approx 2.2 \cdot 10^{-13} \mathrm{erg}\right)$, из $\quad$ (6) получим $\tau_{\mathrm{ep}}(16.400 \mathrm{~K}) \approx 445 \mathrm{~ns}$. Тогда время электронэлектронного рассеяния $\tau_{\mathrm{ee}}$, найденное из формулы $\tau_{\varepsilon}^{-1}=\tau_{\mathrm{ep}}^{-1}+\tau_{\mathrm{ee}}^{-1}[24]$, будет иметь тот же порядок, что и $\tau_{\varepsilon}$, т.е. основной вклад в рассеяние вносит электрон-электронное взаимодействие, как и в работе [24],

Зная параметр $B_{T}$, можно оценить коэффициент теплоотвода $h$ из пленки в подложку в модели разогрева квазичастиц [19]: $h=B_{T} k_{\mathrm{B}} /\left(0.374 c e_{0} R_{\mathrm{sq}} \tau_{\varepsilon}\right)$. Здесь $B_{T}=1000 \mathrm{G}, c=3 \cdot 10^{10} \mathrm{~cm} / \mathrm{s}$ - скорость света, $R_{\mathrm{sq}} \approx 2.8 \cdot 10^{-11} \mathrm{~s} / \mathrm{cm}-$ сопротивление на квадрат. Тогда $h \approx 0.1 \mathrm{~W} /\left(\mathrm{cm}^{2} \cdot \mathrm{K}\right)$.

Альтернативным методом определения $\tau_{\varepsilon}$ является непосредственное наблюдение скачка напряжения на вольт-амперной характеристике образца в некотором магнитном поле [22] и определение напряжения начала неустойчивости Ларкина-Овчинникова $V_{\mathrm{LO}}$. Скорость 
движения вихрей в этой точке определяется формулой [18]:

$$
\begin{aligned}
v_{\mathrm{LO}} & =\left[D(14 \xi(3))^{1 / 2}\left(1-T_{0} / T_{c}(B)\right)^{1 / 2} /\left(\pi \tau_{\varepsilon}\right)\right]^{1 / 2} \\
& \approx\left[1.306\left(D / \tau_{\varepsilon}\right)\left(1-T_{0} / T_{c}(B)\right)^{1 / 2}\right]^{1 / 2}
\end{aligned}
$$

С другой стороны та же скорость может быть найдена экспериментально из формулы [22]

$$
v_{\mathrm{LO}}=V_{\mathrm{LO}} /(B b)
$$

где $b$ - расстояние между потенциальными контактами.

Из формул (7) и (8), можно найти $\tau_{\varepsilon}$.

Так как измерения проводились в полях, близких к критическим, необходимо заметить, что с увеличением поля отношение $V_{\mathrm{LO}} / V_{\mathrm{N}}$ почти не меняется $\left(V_{\mathrm{LO}} / V_{\mathrm{N}} \approx 0.1, V_{\mathrm{N}}\right.$ - напряжение на образце непосредственно после скачка) или стремится к единице [22,23]. В любом случае для оценки можно принять $V_{\mathrm{LO}} \approx V_{\mathrm{N}}$. В нашем случае $b \approx 0.2 \mathrm{~cm}, V_{\mathrm{N}} \approx 1 \mathrm{mV}, B \approx 0.02 \mathrm{~T}$, $T_{0} / T_{c}(B) \approx T_{0} / T_{c 0}=0.9953$. Тогда $\tau_{\varepsilon} \approx 1.7 \mathrm{~ns}$.

\section{5. Заключение}

Температурные и магнитополевые зависимости критического тока перехода в нормальное состояние тонких пленок $\mathrm{NbN}$, исследованные в данной работе, определяются возникновением нормального домена и разогревом электронов при вязком течении магнитного потока. Время энергетической релаксации, найденное в модели разогрева квазичастиц (порядка наносекунды), близко к значениям, полученным другими авторами.

\section{Список литературы}

[1] A. Shurakov, Y. Lobanov, G. Goltsman. Supercond. Sci. Technol. 29, 023001 (2016).

[2] K. Smirnov, A. Divochiy, Yu. Vakhtomin, P. Morozov, Ph. Zolotov, A. Antipov, V. Seleznev. Supercond. Sci. Technol. 31, 035011 (2018).

[3] T. Wakamura, H. Akaike, Y. Omori, Y. Niimi, S. Takahashi, A. Fujimaki, S. Maekawa, Y. Otani. Nature Mater. 14, 675 (2015).

[4] J. Yong, T.R. Lemberger, L. Benfatto, K. Ilin, M. Siegel. Phys. Rev. B 87, 184505 (2013).

[5] J. Jesudasan, M. Mondal, M. Chand, A. Kamlapure, S. Kumar, G. Saraswat, V.C. Bagwe, V. Tripathi, P. Raychaudhuri. AIP Conf. Proc. 1349, 923 (2011).

[6] Н.Д. Кузьмичев, Г.П. Мотулевич. ЖЭТФ 84, 6, 2316 (1983).

[7] R. Jha, A. Kumar, V.P.S. Awana. AIP Conf. Proc. 1447, 867 (2012).

[8] М.А. Васютин, А.И. Головашкин, Н.Д. Кузьмичев. ФТТ 48, 12, 2128 (2006).

[9] N.D. Kuzmichev, M.A. Vasyutin, A.I. Golovashkin. Physica C 460-462, part 2. 849 (2007).

[10] М.А. Васютин, А.И. Головашкин, Н.Д. Кузьмичев. Кратк. сообщ. по физике 9, 48 (2008).
[11] Д.А. Балаев, С.В. Семенов, М.И. Петров. ФТТ 55, 12, 2305 (2013).

[12] R. Matsunaga et al. Science 345, 1145 (2014).

[13] D. Sherman, U.S. Pracht, B. Gorshunov, S. Poran, J. Jesudasan, M. Chand, P. Raychaudhuri, M. Swanson, N. Trivedi, A. Auerbach, M. Scheffler, A. Frydman, M. Dressel. Nature Phys. 11, 188 (2015).

[14] C. Carbillet, S. Caprara, M. Grilli, C. Brun, T. Cren, F. Debontridder, B. Vignolle, W. Tabis, D. Demaille, L. Largeau, K. Ilin, M. Siegel, D. Roditchev, B. Leridon. Phys. Rev. B 93, 144509 (2016).

[15] Z. Wang, A. Kawakami, Y. Uzawa, B. Komiyama. J. Appl. Phys. 79, 7837 (1996).

[16] М.А. Васютин, Н.Д. Кузьмичев, Д.А. Шилкин. ФТТ 58, 2 , 231 (2016).

[17] А.И. Безуглый. ФНТ 26, 8, 755 (2000).

[18] А.И. Ларкин, Ю.Н. Овчинников. ЖЭТФ 68, 5, 1915 (1975).

[19] A.I. Bezuglyi, V.A. Shklovskij. Physica C 202, 234 (1992).

[20] А.Г. Калимов. Физические основы сверхпроводимости. СПб (2007). С. 92.

[21] А.А. Абрикосов. Основы теории металлов. Наука, М. (1987). C. 301.

[22] S.-Z. Lin, O. Ayala-Valenzuela, R.D. McDonald, L.N. Bulaevskii, T.G. Holesinger, F. Ronning, N.R. Weisse-Bernstein, T.L. Williamson, A.H. Mueller, M.A. Hoffbauer, M.W. Rabin, M.J. Graf. Phys. Rev. B 87, 184507 (2013).

[23] Г.В. Самсонов. Нитриды. Наук. думка, Киев (1969). С. 181.

[24] W. Klein, R.P. Huebener, S. Gauss, J. Parisi. J. Low Temp. Phys. 61, 413 (1985).

Редактор К.В. Емцев 